\title{
Emergence of ceftazidime-avibactam-resistant Klebsiella pneumoniae during treatment, Finland, December 2018
}

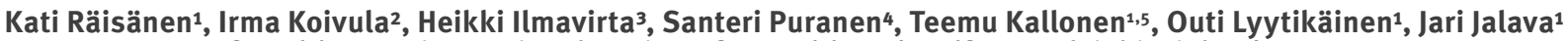

1. Department of Health Security, National Institute for Health and Welfare, Helsinki, Finland

2. Kuopio University Hospital, Unit of Infections and Hospital hygiene, Kuopio University Hospital, Kuopio, Finland

3. Eastern Finland laboratory Centre, Kuopio, Finland

4. Aalto University, Department of Computer Science, Espoo, Finland

5. Department of Biostatistics, University of Oslo, Oslo, Norway

Correspondence: Kati Räisänen (kati.raisanen@thl.fi)

Citation style for this article:

Räisänen Kati, Koivula Irma, Ilmavirta Heikki, Puranen Santeri, Kallonen Teemu, Lyytikäinen Outi, Jalava Jari. Emergence of ceftazidime-avibactam-

resistant Klebsiella pneumoniae during treatment, Finland, December 2018. Euro Surveill. 2019;24(19):pii=1900256. https://doi.org/10.2807/1560-7917.

ES.2019.24.19.1900256

Article submitted on 24 Apr 2019 / accepted on 07 May 2019 / published on 09 May 2019

In December 2018, a ceftazidime-avibactam (CAZAVI)-resistant KPC-2-producing Klebsiella pneumoniae strain was isolated in Finland. CAZ-AVI resistance was observed 34 days after CAZ-AVI treatment in a trauma patient transferred from a hospital in Greece who had been colonised with $\mathrm{bla}_{\mathrm{KPC}-2}$-producing $K$. pneumoniae $\mathrm{ST}_{39}$, and later developed a bloodstream infection. The CAZ-AVI-resistant strain contained a novel 15 amino acid insertion in the KPC-2 protein causing structural changes proximal to the KPC-2 active site.

Ceftazidime-avibactam (CAZ-AVI) is a promising novel $\beta$-lactam- $\beta$-lactamase inhibitor combination with activity against multidrug-resistant (MDR) Enterobacteriaceae for which there are limited treatment options, e.g. those producing $K$. pneumoniae carbapenemases (KPC). According to the rapid risk assessment published by the European Centre for Disease Prevention and Control (ECDC) in June 2018, the emergence of CAZ-AVI resistance in Europe is a very rare phenomenon but an important cross-border threat that should be monitored carefully [1]. Here, we report the emergence of CAZ-AVI resistance in a $\mathrm{KPC}$-2-producing $K$. pneumoniae strain and describe a mutation associated with the resistance.

\section{Case description}

Following a traffic accident, a patient without any known underlying diseases was hospitalised in Greece. The patient was first admitted to a local hospital before being transferred to a tertiary hospital 3 days later; 27 days after admittance the patient was moved to a university hospital in Finland. There was no information on carriage of carbapenemase-producing Enterobacteriaceae (CPE) in the medical notes received from the Greek hospital. The screening specimens for MDR bacteria were obtained at admission to the
Finnish hospital according to the national guidelines [2] including rectal swabs and skin lesions. The case was isolated and treated with contact precautions. The antimicrobials (metronidazole, vancomycin, tigecycline and colistin) that had been prescribed in Greece were stopped after admittance since the patient showed no clinical signs or symptoms of infection.

The screening specimen from sacrum decubitus was positive for KPC-2-producing $K$. pneumoniae sequence type (ST)39 (isolate 1), which was susceptible to CAZ$\mathrm{AVI}$ and resistant to carbapenems and several other antimicrobials (Table). The patient developed fever 16 days after admission to the hospital in Finland. Treatment with tigecyclin and CAZ-AVI was initiated after obtaining blood cultures, which yielded KPC-2producing $K$. pneumoniae ST39 (isolate 2), susceptible to CAZ-AVI and resistant to carbapenems. The treatment with tigecyclin and CAZ-AVI was administered for 2 weeks. After 2 days without antimicrobials, the patient developed fever again and treatment with CAZ$\mathrm{AVI}$ and fosfomycin was initiated and continued for 19 days. Ten days after treatment ended, the patient developed fever once more. Blood cultures were found to be positive for KPC-2-producing K. pneumoniae ST39 (isolate 3), resistant to both CAZ-AVI and carbapenems but sensitive to sulfamethoxazole-trimethoprim. Colistin and sulfamethoxazole-trimethoprim were administered for 12 days. The patient subsequently recovered from the infection.

\section{Molecular characterisation of CAZ-AVI resistance mechanism}

All three KPC-2-producing K. pneumoniae $\mathrm{ST} 39$ isolates were characterised by whole genome sequencing method and subsequent data by core-genome multilocus sequence typing (cgMLST) as previously described [3]. The isolated strains, with six to 13 allelic differences, 
Antimicrobial susceptibility and genetic characteristics of Klebsiella pneumoniae clinical isolates, Finland, OctoberDecember 2018

\begin{tabular}{|c|c|c|c|c|c|c|c|c|c|}
\hline \multirow{2}{*}{ Isolate } & \multirow{2}{*}{ Type of specimens } & \multicolumn{7}{|c|}{ MIC $(\mu \mathrm{L} / \mathrm{mL})$} & \multirow{2}{*}{$\beta$-lactamase resistance genes } \\
\hline & & ETP & MEM & CAZ-AVI & CST & FOF & SXT & TGC & \\
\hline Isolate 1 & Sacrum, decubitus & $>32$ & $>32$ & 1 & 8 & 64 & $\mathrm{R}^{\mathrm{a}}$ & 1 & bla $_{\text {KPC-2 }}$, bla $_{\text {TEM- }}$, bla $_{\text {SHV }-11}$ \\
\hline Isolate 2 & Blood & $>32$ & $>32$ & $\ll 1$ & $>8$ & 32 & $R^{a}$ & 4 & bla $_{\mathrm{KPC}-2}, \mathrm{bla}_{\mathrm{TEM}-1}$, bla $_{\mathrm{SHV}-11}$ \\
\hline Isolate 3 & Blood & 6 & 16 & $>16$ & 0,5 & $>1,024$ & 0,38 & 1,5 & bla $_{\mathrm{KPC}-2}$ variant $^{\mathrm{b}}, \mathrm{bla}_{\mathrm{SHV}-11}$ \\
\hline
\end{tabular}

CAZ-AVI, Ceftazidime-avibactam; CST, colistin; ETP, ertapenem; FOF, fosfomycin; MEM, meropenem; MIC, minimum inhibitory concentration; SXT, trimethoprim-sulfamethoxazole; TGC, tigecycline.

${ }^{a}$ Only interpretation results of susceptibility testing were available and strains were resistant (R).

${ }^{b} 15$ amino acids insertion (AVYTRAPNKDDKHSE) of KPC-2 variant after position 259.

Susceptibility testing and interpretation were carried out according to the European Committee on Antimicrobial Susceptibility Testing breakpoints [12].

were all categorised as belonging to the same clone according to the cgMLST [4]. The first two (isolates 1 and 2 ) had three $\beta$-lactam resistance genes ( bla $_{\text {TEM-1 }}$, bla $_{\mathrm{SHV}-11}$ and $\mathrm{bla}_{\mathrm{KPC}-2}$ ) and the third (isolate 3 ) had two such genes (bla $\mathrm{SHV}-11_{11}$ and a bla $\mathrm{KPC}-2$ variant). The third isolate had lower minimal inhibition concentrations (MIC) of meropenem and ertapenem than those of the first two isolates. The bla $\mathrm{KPC}-2_{2}$ genes from the first two isolates were identical. The bla $\mathrm{KPC}-2_{2}$ gene from the third isolate had an insertion of 45 nucleotide corresponding to 15 amino acid insertion after position 259 of the KPC-2 protein (GenBank accession number MK823188).

\section{Discussion}

After the ECDC published its rapid risk assessment of the emergence of CAZ-AVI resistance, the National Institute for Health and Welfare (THL) informed clinical microbiology laboratories in Finland and asked them to notify THL of any CAZ-AVI resistant isolates. To our knowledge, this is the first CAZ-AVI-resistant $K$. pneumoniae with bla $_{\mathrm{KPC}}$ gene isolated in Finland and the second isolated in Europe.

CAZ-AVI resistance was observed after 34 days of CAZ-AVI treatment in a patient who was colonised by bla $_{\mathrm{KPC}_{-}}$-producing $K$. pneumoniae ST39 and later developed a blood stream infection. The strain isolated after CAZ-AVI treatment had a mutated bla $_{\mathrm{KPC}-2}$ gene encoding KPC-2 protein with 15 amino acid insertion; the observed mutation in bla $_{\mathrm{KPC}-2}$ gene has not been described previously. Comparative protein modelling $[5,6]$ based an inhibitor-blocked KPC-2 crystal structure (PDB ID: 5 UJ4) reveals that the 15 amino acid insertion adds to a loop region connecting the central beta-sheet to the carboxyl-terminal alpha-helix proximal to the KPC-2 active site.

We hypothesise that the resulting structural change weakens avibactam's inhibitory effect by disrupting its ability to bind at the active site, thereby causing resistance. Mutations in bla $_{\mathrm{KPC}}$ genes have been associated with the development of CAZ-AVI resistance after CAZ$A V I$ treatment in a few occasions. The first report from the United States (US) identified K. pneumoniae ST258 isolates with three mutations in KPC-3 (D179Y/T243M double substitution, D179Y and V240G) after 10-19 days of CAZ-AVI treatment $[7,8]$. The D179Y mutation in KPC-3 was also described in the K. pneumoniae ST1519 strain from Italy where CAZ-AVI resistance arose after 17 days of CAZ-AVI treatment [9]. The second report from the US described the same D179Y mutation in KPC-2 in K. pneumoniae ST258 isolate after 12 days of CAZ-AVI treatment [10]. In these earlier reports, KPC mutations causing CAZ-AVI resistance decreased meropenem MICs and some strains became even susceptible to meropenem. We detected similar decrease in meropenem MICs but our strain remained resistant. In addition to KPC gene mutations, CAZ-AVI resistance has been associated to CTX-M-14 mutations [11].

In conclusion, treating infections caused by KPCproducing $K$. pneumonia with CAZ-AVI can select KPC-variants that cause CAZ-AVI resistance and simultaneously preserve carbapenem resistance. Therefore, careful monitoring of resistance development by bacterial cultures and subsequent susceptibility testing is important.

\section{Acknowledgements}

The authors wish to acknowledge THL Expert Microbiology laboratory for technical assistance, Anne-Mari Rissanen, Eastern Finland laboratory Centre, Kuopio, Finland for microbiological consultation and CSC - IT Center for Science, Finland, for providing computational resources.

\section{Conflict of interest}

None declared.

\section{Authors' contribution}

K. Räisänen: whole genome sequencing and data analysis, preparation of table, manuscript writing. I. Koivula: patient treatment. H. Ilmavirta: detection and characterization of bacterial isolates, resistance typing. S. Puranen: protein modeling. T. Kallonen: data analysis. O. Lyytikäinen: 
manuscript writing. J. Jalava: data analysis, manuscript writing

\section{References}

1. European Centre for Disease Prevention and Control (ECDC). Emergence of resistance to ceftazidime-avibactam in carbapenem-resistant Enterobacteriaceae - 12 June 2018. Stockholm; ECDC; 2018. Available from: https://ecdc.europa. eu/en/publications-data/rapid-risk-assessment-emergenceresistance-ceftazidime-avibactam-carbapenem

2. Julkari. Ohje moniresistenttien mikrobien tartunnantorjunnasta [Help with anti-microbial infection control]. Finland: Julkari; 2017. Finnish. Available from: http://urn.fi/ URN:ISBN:978-952-302-943-9.

3. van Beek J, Räisänen K, Broas M, Kauranen J, Kähkölä A, Laine J, et al. Tracing local and regional clusters of carbapenemaseproducing Klebsiella pneumoniae ST512 with whole-genome sequencing, Finland, 2013-2018. Euro Surveill. 2019; (Forthcoming).

4. Kluytmans-van den Bergh MF, Rossen JW, Bruijning-Verhagen PC, Bonten MJ, Friedrich AW, Vandenbroucke-Grauls CM, et al. Whole-Genome Multilocus Sequence Typing of ExtendedSpectrum-Beta-Lactamase-Producing Enterobacteriaceae. J Clin Microbiol. 2016;54(12):2919-27. https://doi.org/10.1128/ JCM.01648-16 PMID: 27629900

5. Šali A, Blundell TL. Comparative protein modelling by satisfaction of spatial restraints. J Mol Biol. 1993;234(3):779815. https://doi.org/10.1006/jmbi.1993.1626 PMID: 8254673

6. Schrödinger LLC. PyMOL by Schrödinger. Molecular Graphics System. Version 2.1.0. Available from: https://pymol.org

7. Shields RK, Chen L, Cheng S, Chavda KD, Press EG, Snyder A, et al. Emergence of Ceftazidime-Avibactam Resistance Due to Plasmid-Borne blaKPC-3 Mutations during Treatment of Carbapenem-Resistant Klebsiella pneumoniae Infections. Antimicrob Agents Chemother. 2017;61(3):e02097-16. https:// doi.org/10.1128/AAC.02097-16 PMID: 28031201

8. Haidar G, Clancy CJ, Shields RK, Hao B, Cheng S, Nguyen MH. Mutations in blaKPC-3 That Confer Ceftazidime-Avibactam Resistance Encode Novel KPC-3 Variants That Function as Extended-Spectrum $\beta$-Lactamases. Antimicrob Agents Chemother. 2017;61(5):e02534-16. https://doi.org/10.1128/ AAC.02534-16 PMID: 28223379

9. Gaibani P, Campoli C, Lewis RE, Volpe SL, Scaltriti E, Giannella $M$, et al. In vivo evolution of resistant subpopulations of KPC-producing Klebsiella pneumoniae during ceftazidime/ avibactam treatment. J Antimicrob Chemother. 2018;73(6):15259. https://doi.org/10.1093/jac/dkyo82 PMID: 29566151

10. Giddins MJ, Macesic N, Annavajhala MK, Stump S, Khan S, Mcconville TH, et al. Successive Emergence of CeftazidimeAvibactam Resistance through Distinct Genomic Adaptations in blaKPC-2-Harboring Klebsiella pneumoniae Sequence Type 307 Isolates. 2018;62(3):e02101-17.

11. Both A, Büttner H, Huang J, Perbandt M, Belmar Campos $C$, Christner $M$, et al. Emergence of ceftazidime/avibactam non-susceptibility in an MDR Klebsiella pneumoniae isolate. J Antimicrob Chemother. 2017;72(9):2483-8. https://doi. org/10.1093/jac/dkx179 PMID: 28637339

12. The European Committee on Antimicrobial Susceptibility Testing (EUCAST). Breakpoint tables for interpretation of MIC and zone diameters. Version 8.1, 2018. Växjö: EUCAST; 2018. Available from: http://www.eucast.org.

\section{License, supplementary material and copyright}

This is an open-access article distributed under the terms of the Creative Commons Attribution (CC BY 4.0) Licence. You may share and adapt the material, but must give appropriate credit to the source, provide a link to the licence and indicate if changes were made.

Any supplementary material referenced in the article can be found in the online version.

This article is copyright of the authors or their affiliated institutions, 2019. 